\title{
Using Time-Delay Neural Network (DTDNN) for Enhancement Power Systems Dynamic Stability
}

\author{
Abir Aljarbouh \\ Post graduate Student (PhD) \\ Faculty of Electrical \& Electronics \\ Engineering \\ University of Aleppo/Syria
}

\author{
Ahmad Sharam \\ Assistant Professor \\ Faculty of Electrical \& Electronics \\ Engineering \\ University of Aleppo/Syria
}

\author{
Mohamad Khateeb \\ Assistant Professor \\ Artificial Intelligent, Faculty of \\ Information Engineering, \\ University of Aleppo/Syria
}

\begin{abstract}
The design of two level power system stabilizer (PSS) controller is discussed in this article. First level is conventional PSS controller, while the second level is designed using following two methods: New Coordinated Fuzzy-PID controller and Distributed Time-Delay Neural Network (DTDNN) controller, Speed deviation and derivative of speed deviation of synchronous generator are taken as the input to the controller and voltage signal is the output of the controller.
\end{abstract}

The main function of the conventional power system stabilizers is to enhance the damping of low frequency oscillations in power system, while the power system stabilizer which is designed using Fuzzy-PID and distributed time-delay neural network improves the total dynamic response of power system to achieve the required results

This technique is applied on a single machine infinite bus (SMIB) power system. The distributed time-delay neural network damps out the low frequency oscillations and enhances the power system dynamic stability in the better manner than the conventional power system stabilizer.

\section{Keywords}

Fuzzy-PID- Distributed Time-Delay Neural Network (DTDNN)- frequency oscillations.

\section{INTRODUCTION}

The rapid development of power systems and increasingly diverse problems, demands a real-time settlement. Therefore, this paper proposes the design of power system stabilizers (PSS) based on Distributed Time-Delay Neural Network (DTDNN) that can respond to changes in system performance directly. In this study DTDNN PSS was applied on a single machine system. The application of DTDNN PSS on a single machine system is emphasized on DTDNN PSS performance against low frequency oscillations and improved performance of system.

Enhancement power systems stability and damping oscillations and enhancement the dynamic performance of power systems by controlling the excitation system using a power regulators (PSS: power system stabilizers) which provides good damping. These dampers have a simple structure, flexibility and simple implementation, but due to the large non-linear characteristics of the power system, the PSS cannot provide acceptable performance, especially when large changes in operating conditions [1], in addition to that the gains of the controllers must be determined for special operating conditions and thus These controllers are not responsive to new operating conditions [2].
The fuzzy logic controller can handle well with complex nonlinear systems, and it also does not need exact knowledge of the mathematical model of the studied system and it is programmed with linguistic rules with the general structure of the if-then rule, which can be developed depending on experience Specialists and experts [3], this contribution has been developed of a new coordinated Fuzzy-PID controller for a SMIB power system. To show the robustness and effectiveness of this coordinate under all disturbance cases than other convention controllers for all study cases.

\section{SYSTEM CONFIGURATION}

\subsection{The Power System Model}

Figure 1 show the studied system which is a Single Machine Infinite Bus (SMIB) [4]. The model used for representing the synchronous generator connected through transmission line to a very large power network by an infinite bus. The synchronous generator is driven by a turbine with a governor and excited by an external excitation system [5].

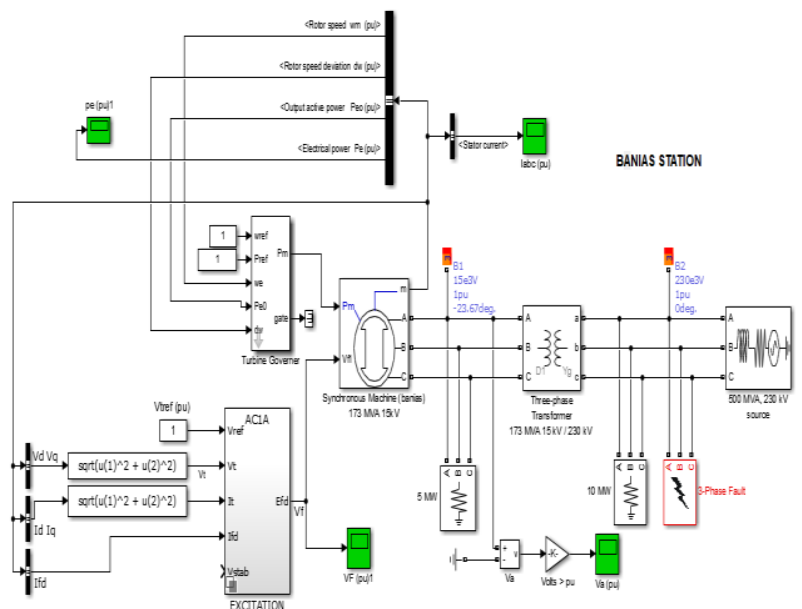

Figure 1: synchronous generator model in the MATLAB / SIMULINK.

The station generator was represented by a sixth degree model by the electromagnetic field of the state sub-transit $E_{q}^{\prime \prime}, E_{d}^{\prime \prime}$ and its impedance $X_{q}^{\prime \prime}, X_{d}^{\prime \prime}$ the electromagnetic oscillation equation and the electric power equation as follows:

$\left[\begin{array}{l}v_{d} \\ v_{q}\end{array}\right]=\left[\begin{array}{l}E_{d}^{\prime \prime} \\ E_{q}^{\prime \prime}\end{array}\right]-\left[\begin{array}{cc}R & x_{q}^{\prime \prime} \\ -x_{d}^{\prime \prime} & R\end{array}\right]\left[\begin{array}{l}i_{d} \\ i_{q}\end{array}\right]$

$\frac{d \Delta \omega}{d t}=\frac{1}{M}\left(P_{m}-P_{e}-D \Delta \omega\right)$ 
$\Delta \omega=\omega-\omega_{s}=\frac{d \delta}{d t}$

$T_{d o}^{\prime} E_{q}^{\prime}=E_{f}-E_{q}^{\prime}+i_{d}\left(x_{d}-x_{d}^{\prime}\right)$

$T_{q o}^{\prime} E_{d}^{\prime}=-E_{d}^{\prime}+i_{d}\left(x_{q}-x_{q}^{\prime}\right)$

$T_{d o}^{\prime \prime} E_{q}^{\prime \prime}=E_{q}^{\prime}-E_{q}^{\prime \prime}+i_{d}\left(x_{d}^{\prime}-x_{d}^{\prime \prime}\right)$

$T_{q o}^{\prime \prime} E_{d}^{\prime \prime}=E_{d}^{\prime}-E_{d}^{\prime \prime}+i_{q}\left(x_{q}^{\prime}-x_{q}^{\prime \prime}\right)$

It represents $E_{q}, E_{q}^{\prime}, E_{q}^{\prime \prime}$ excitation voltage and $T_{q o}^{\prime}, T_{d o}^{\prime}$ time constant of the open circuit field, and $X_{d}, X_{d}^{\prime}, X_{d}^{\prime \prime}$ impedance transient impedance, and sub-transient impedance respectively for the generator towards the direct axis $\mathrm{d}$, and $X_{q}, X_{q}^{\prime}, X_{q}^{\prime \prime}$ impedance, transient impedance, and sub-transient impedance respectively for the generator towards the quadrature axis q.

\section{CONVENTIONAL CONTROLLER MODEL}

\subsection{Power System Stabilizer Model (PSS)}

The capacity of a power system to maintain stability depends largely on the power stabilizers or Power System Stabilizer (PSS). The function of the PSS is to damp the oscillations of the generator. It acts on the excitation of the machine [6]. The conventional controller PSS acts on the excitation system of the synchronous machine, this controller has two inputs (the speed variation $\Delta \omega$ and electrical power $\mathrm{Pe}$ ) and an output Vpss, which constitutes the input to the excitation system as shown in Figure 2. The performance of a conventional controller is limited. The instrument is sensitive to changes in power system parameters [7].

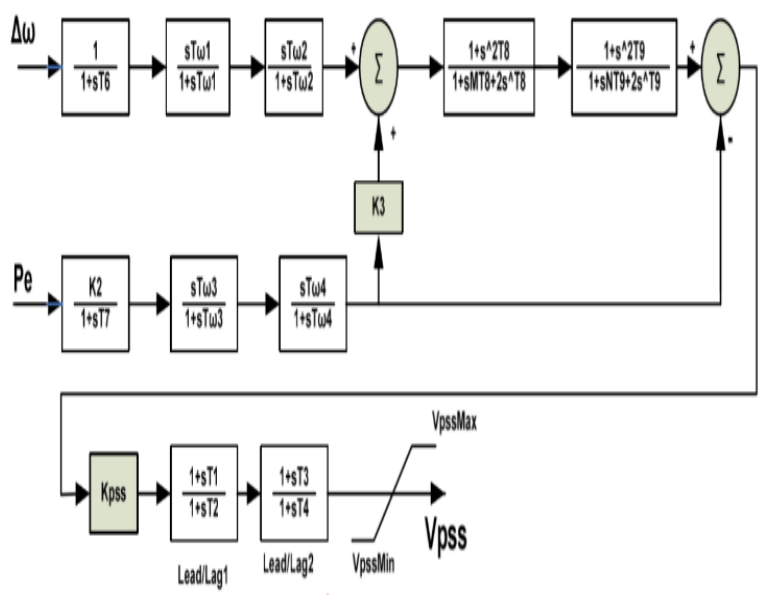

Fig 2: conventional controller PSS model

The transfer functions of the conventional Power System Stabilizer(PSS):

$\operatorname{Vpss}=G F \times\left(K s 1 \times \frac{1+\operatorname{sTlead}(1,3)}{1+\operatorname{sTlag}(2,4)}\right)$

$G F=\left\{\left(\frac{1}{1+s T_{6}} \times \frac{s T_{W 1}}{1+s T_{W 1}} \times \frac{s T_{W 2}}{1+s T_{W 2}}\right) \Delta \omega+\left(\frac{K_{2}}{1+s T_{7}} \times \frac{s T_{W 4}}{1+s T_{W 4}} \times\right.\right.$

$\left.\left.\frac{s T_{W 3}}{1+s T_{W 3}} \times K_{3}\right) P_{e}\right\} \times\left[\left(\frac{1+s^{2} T_{8}}{1+s M T_{8}+2 s^{2} T_{8}} \times \frac{1+s^{2} T_{9}}{1+s M T_{9}+2 s^{2} T_{9}}\right)-\right.$

$\left.P_{e}\left(\frac{K_{2}}{1+s T_{7}} \times \frac{s T_{W 4}}{1+s T_{W 4}} \times \frac{s T_{W 3}}{1+s T_{W 3}}\right)\right]$

\subsection{PID Controller}

The proportional integral and derivative (PID) controller is a one of the earliest industrial controllers. It has many advantages: Its cost is economic, simple easy to be tuned and robust [8] [9] [10]. The practical PID structure is illustrated in Figure 3.
In the parallel form of the PID controller, there are three simple gains $\boldsymbol{K}_{\boldsymbol{P}}, \boldsymbol{K}_{\boldsymbol{I}}$ and $\boldsymbol{K}_{\boldsymbol{D}}$ are used in the decoupled branches of the PID controller which tuned by using PID tuner in Simulink model. The term $\left(\frac{\mathbf{1}}{\tau s+1}\right)$ acts as an effective low-pass filter on the D regulator to attenuate noise in the derivative block [9].

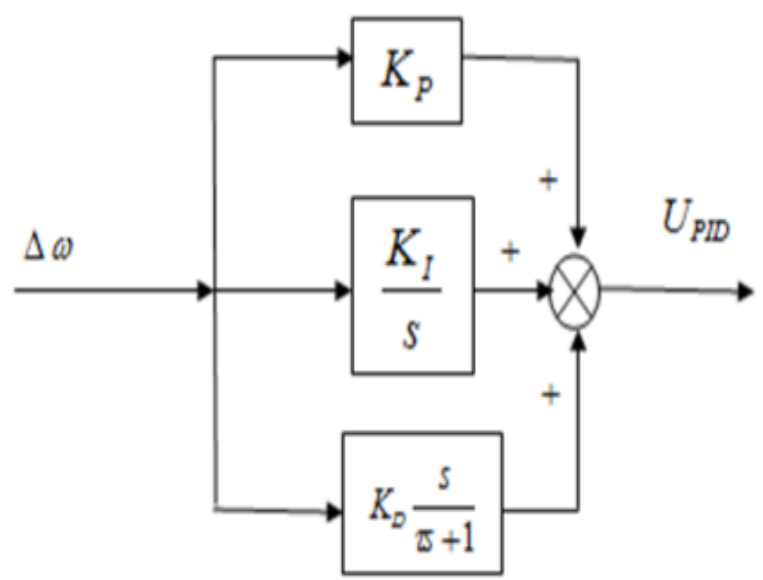

Fig 3: Structure of PID_PSS

\subsection{Fuzzy controller systems}

Since the concept of fuzzy logic given by zadeh in 1965, it has found application in various area including a controller for PSS. A fuzzy controller as shown in fig 4 comprises of four stages: fuzzification, a knowledge base, decision making, and defuzzification. The fuzzification interface coverts input data into suitable linguistic values that can be viewed as label fuzzy sets. The knowledge base comprises knowledge of application domain and attendant control goals by means of set of linguistic control rules. The decision making is the aggregation of output of various control rules that simulate the capability of human decision making. The defuzzification interface performs scale mapping, which converts the range of values of output variables into corresponding universe of discourse.

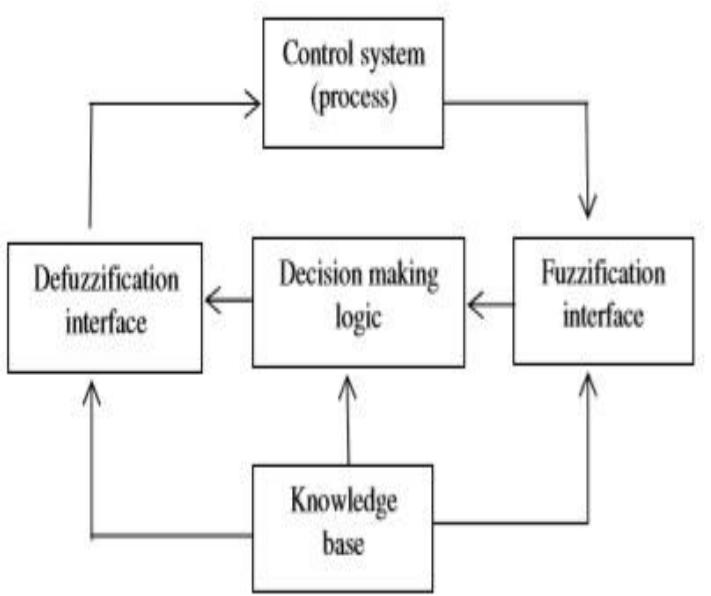

Fig. 4: The basic structure of the fuzzy controller

The fuzzy controller used in PSS is normally a two input and a single output component. The design of fuzzy logic based PSS shown in fig 5. The two inputs are change in angular speed (speed deviation) and rate of change of angular speed (acceleration speed) whereas output of fuzzy logic controller is a voltage signal (V-FLPSS). 


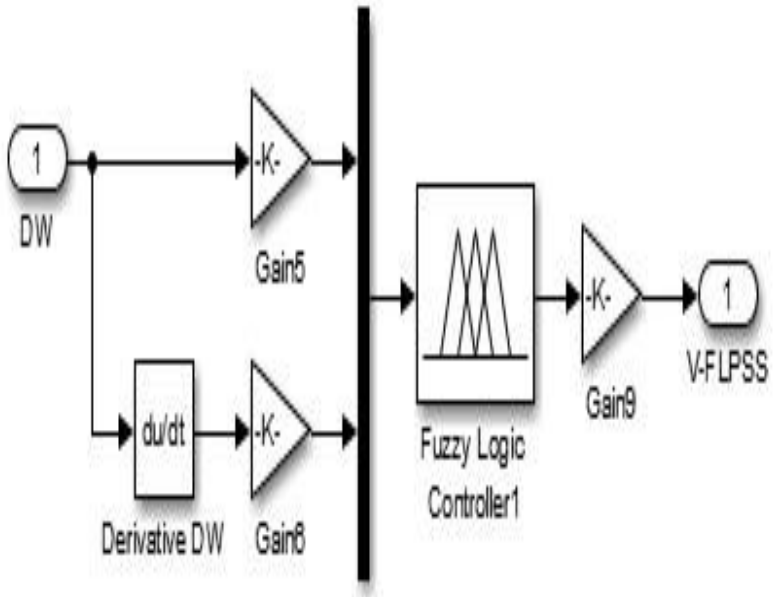

Fig 5: Structure of Fuzzy-PSS

The design starts with assigning the mapped variable inputs/output of the fuzzy logic controller (FLC). The first input variable to the FLC is the generator speed deviation and the second is acceleration. The output variable to the FLC is the voltage. After choosing proper variables as input and output of fuzzy controller, it is required to decide on the linguistic variables. These variables transform the numeric values of the input of fuzzy controller to fuzzy quantities. The number of linguistic variables describing the fuzzy subsets of a variable varies according to the application. Here seven linguistic variables for each of the input and output variables are used to describe them. The membership functions (MF) maps the crisp values into fuzzy variables. The triangular membership functions are used to define the degree of membership. Here for each input variable, seven labels are defined namely, NB, NM, NS, Z, PS, PM and PB. Each subset is associated with triangular membership function to form a set of seven membership functions for each variable, the membership function for speed deviation, acceleration and voltage are shown in figs 8,9,10 respectively, Knowledge base involves defining the rules represented as IF-THEN rules statements governing the relationships between input and output variables in terms of membership functions.

Table.1 Membership functions for fuzzy variables

\begin{tabular}{|c|c|}
\hline NB & Negative Big \\
\hline NM & Negative Medium \\
\hline NS & Negative Small \\
\hline$Z$ & zero \\
\hline PS & Positive Small \\
\hline PM & Positive Medium \\
\hline PB & Positive Big \\
\hline
\end{tabular}

In this stage the input variables speed deviation and acceleration are processed by the inference engine that execute $7 \times 7$ rules represented in rule fig 6 Each rule conjuncts speed deviation $(\omega)$ and acceleration fuzzy set values. The knowledge required to generate the fuzzy rules can be derived from offline simulation. Some knowledge can be based on the understanding of the behavior of the dynamic system under control. For monotonic systems, a symmetrical rule table is very appropriate, although sometimes it may need slight adjustment based on the behavior of the specific system.
If the system dynamics are not known are highly nonlinear, trial and error procedures and experience play an important role in defining the rules.

\begin{tabular}{|l|l|l|l|l|l|l|l|}
\hline \multirow{2}{*}{ DW } & \multicolumn{7}{|c|}{$\mathrm{d}(\mathrm{dw}) / \mathrm{dt}$} \\
\cline { 2 - 8 } & NB & NM & NS & Z & PS & PM & PB \\
\hline NB & NB & NB & NB & NB & NM & NS & Z \\
\hline NM & NB & NB & NM & NM & NS & Z & PS \\
\hline NS & NB & NM & NS & NS & Z & PS & PM \\
\hline Z & NM & NS & Z & Z & PM & PB & PB \\
\hline PS & NM & NS & PS & PM & PM & PB & PB \\
\hline PM & NS & Z & PS & PM & PM & PB & PB \\
\hline PB & Z & Z & PM & PB & PB & PB & PB \\
\hline
\end{tabular}

Fig 6: Decision table of 49 rules for (Fuzzy_PSS)

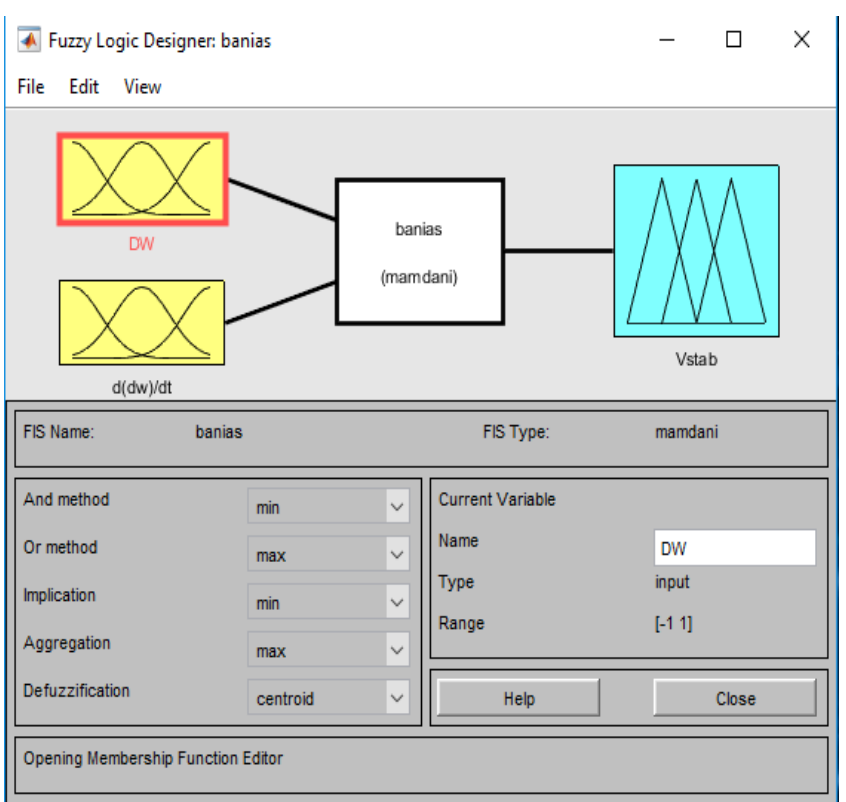

Fig 7: Structure of (Fuzzy_PSS) designer

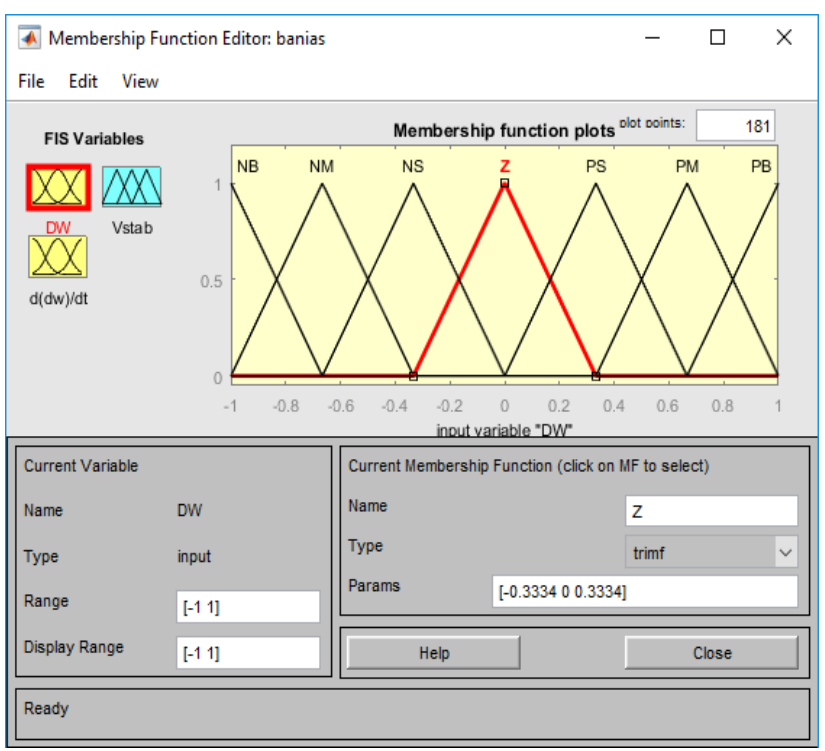

Fig 8: Membership function editor for (DW) 


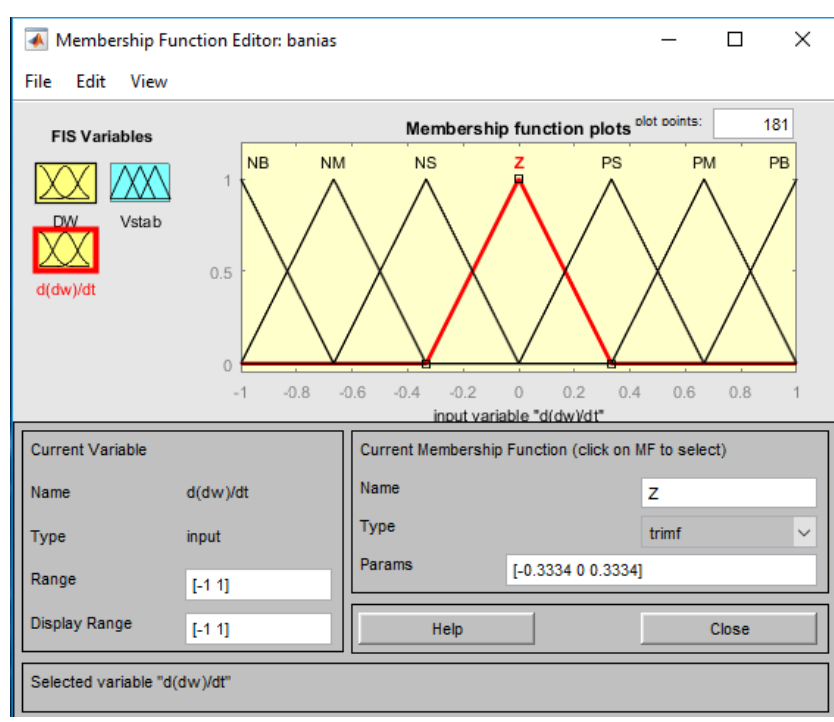

Fig 9: Membership function editor for $(\mathbf{d}(\mathbf{d w}) / \mathrm{dt})$

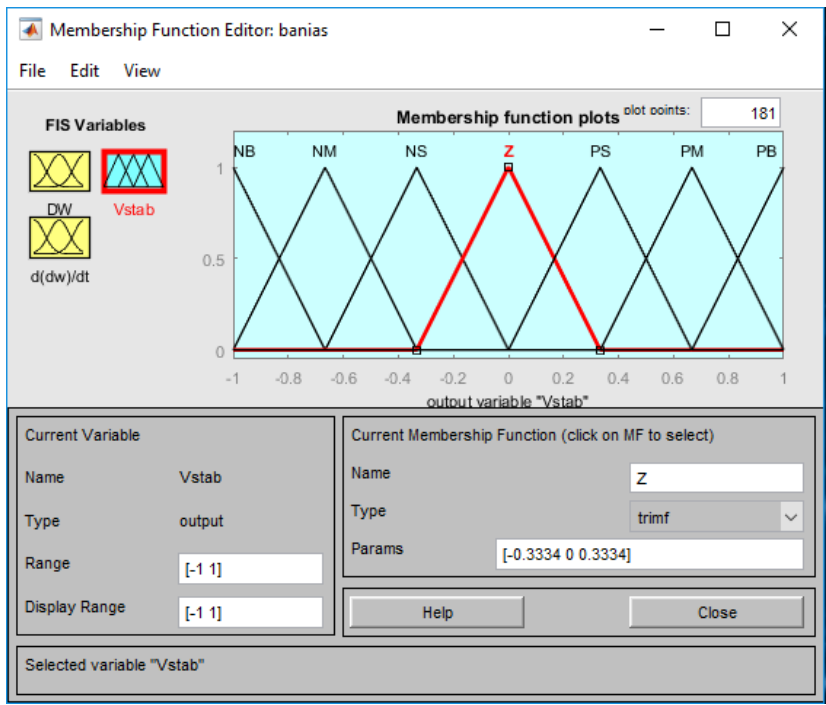

Fig 10: Membership function editor for (Vstab)

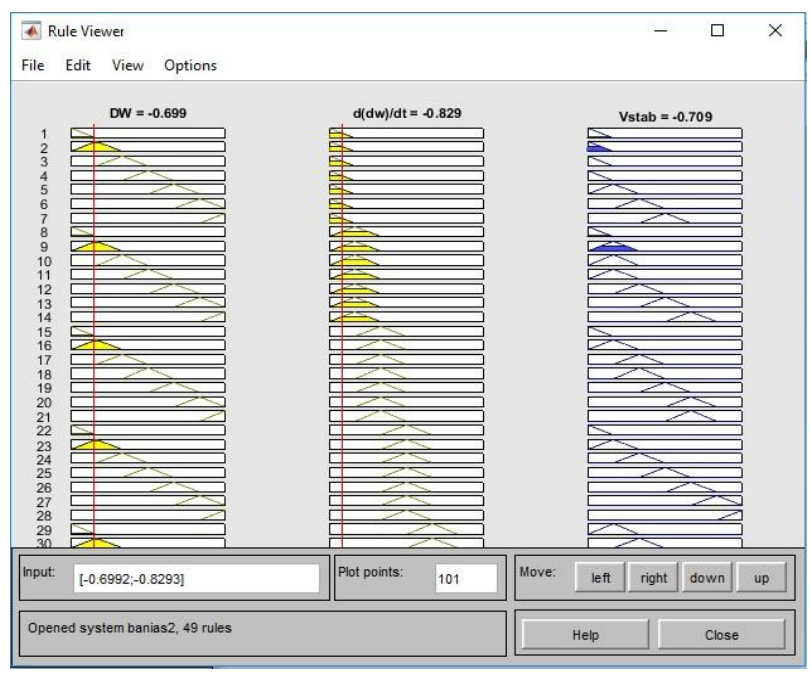

Fig 11: Rule Viewer of (Fuzzy_PSS)

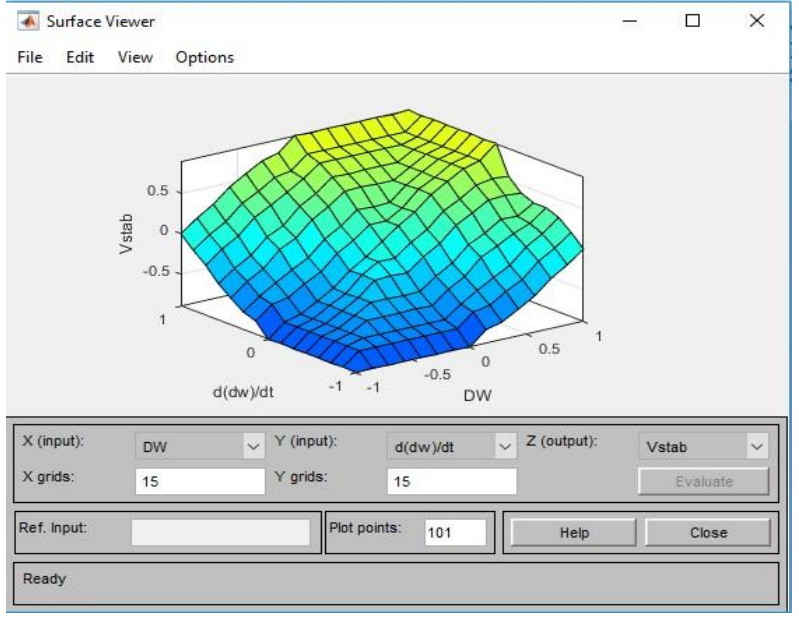

Fig 12: Surface Viewer Rule Viewer of (Fuzzy_PSS)

\subsection{Design of Fuzzy-PID Controller:}

The proposed coordinated of Fuzzy-PID controller block diagram is given in Figure 13. Speed deviation and acceleration have been selected as controller inputs, and the output of the coordination (Fuzzy-PID) is injected into voltage summing point. Inputs and output values which are speed deviation, acceleration inputs and reference voltage output lies between $[-1: 1]$ and we adjust inputs factors Gain5, Gain6 to normalize inputs and then we change Gain 9 to obtain optimal result [11]. Designing the coordinated of (Fuzzy-PID_PSS) depends on designing FLC and PID, tuning PID controller by using PID tuner in Matlab/Simulink model.

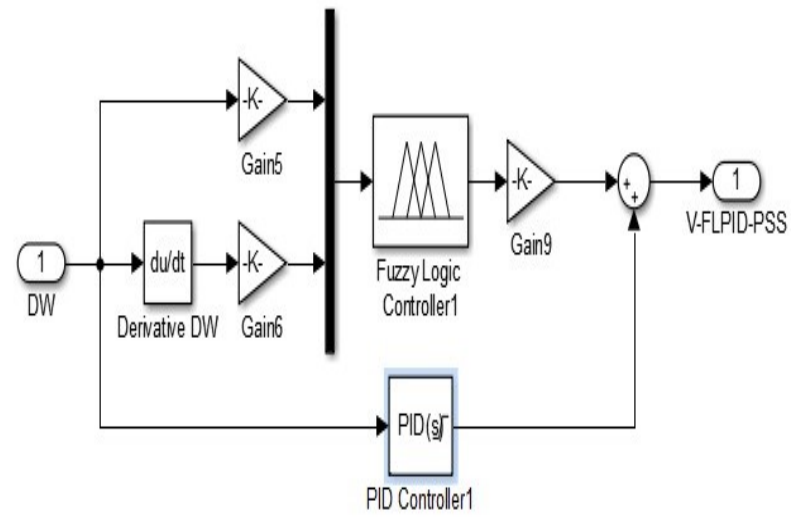

Fig 13: Structure of (Fuzzy-PID_PSS)

\section{PSS DESIGN USING DISTRIBUTED TIME-DELAY NEURAL NETWORK (DTDNN):}

Artificial neural networks are typically organized in layers: the input layer, hidden layer and output layer, which consist of several neurons. Each neuron in a layer is connected to adjacent layers by weights. Data are presented to the network via the input layer, which are multiplied by the weights, and then go through the activation function of the neuron in one or more hidden layers. The function in the output layer computes the output of the artificial neuron [12]. DTDNN is it dynamic networks are generally more powerful than static networks because dynamic networks have memory, they can be trained to learn sequential or time varying patterns. In dynamic networks, the output depends not only on the current input to the network, but also on the current or previous inputs, 
outputs, or states of the network. In this article, each layer in the Distributed Time-Delay Artificial Neural Network is made up of the following parts:

- Set of weight matrices that come into that layer (which can connect from other layers or from external inputs), associated weight function rule used to combine the weight matrix with its

- input (normally standard matrix multiplication), and associated tapped delay line.

- Bias vector.

- Net input function rule that is used to combine the outputs of the various weight functions with the bias to produce the net input (normally a summing junction).

- Transfer function.

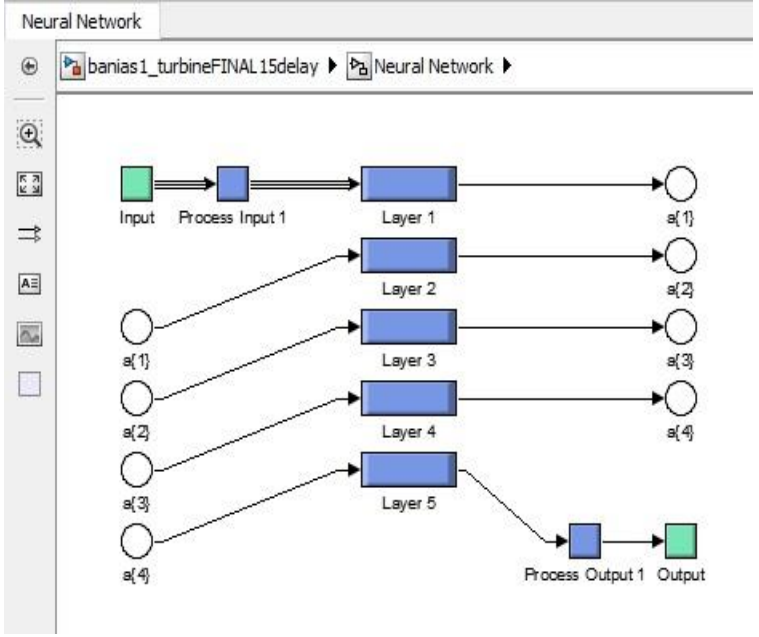

Fig14: Layers of Suggested (DTDNN) Neural Network

The network has inputs that are connected to special weights, called input weights, and denoted by IWij, where $\mathrm{j}$ denotes the number of the input vector that enters the weight, and $\mathrm{i}$ denotes the number of the layer to which the weight is connected. The weights connecting one layer to another are called layer weights and are denoted by Wij, where $\mathrm{j}$ denotes the number of the layer coming into the weight and i denotes the number of the layer at the output of the weight Fig 15

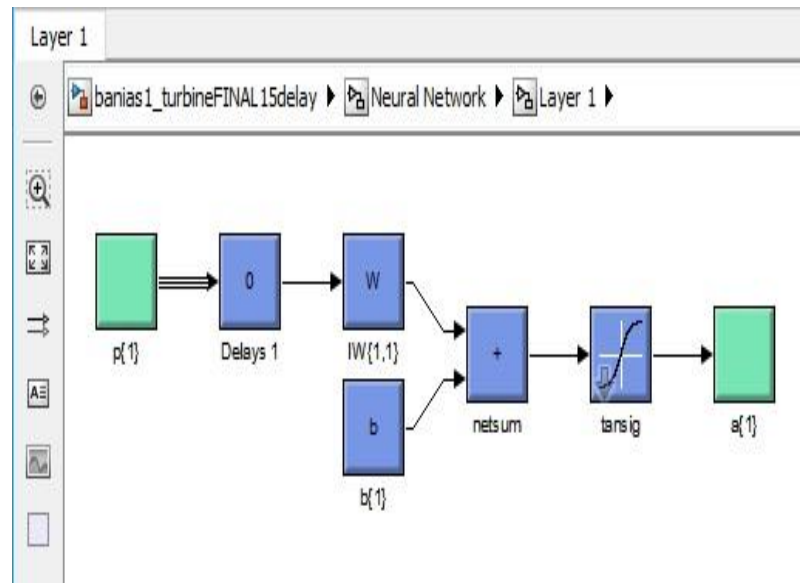

Fig 15: Structure Layer1 of (DTDNN) neural network

The network structure used in this training consists of several layers, namely the input layer, the hidden layers and the output layer, the first fourth layers contain 20 neurons while the fifth layer contains one neuron, Fig 14 and Fig 16.

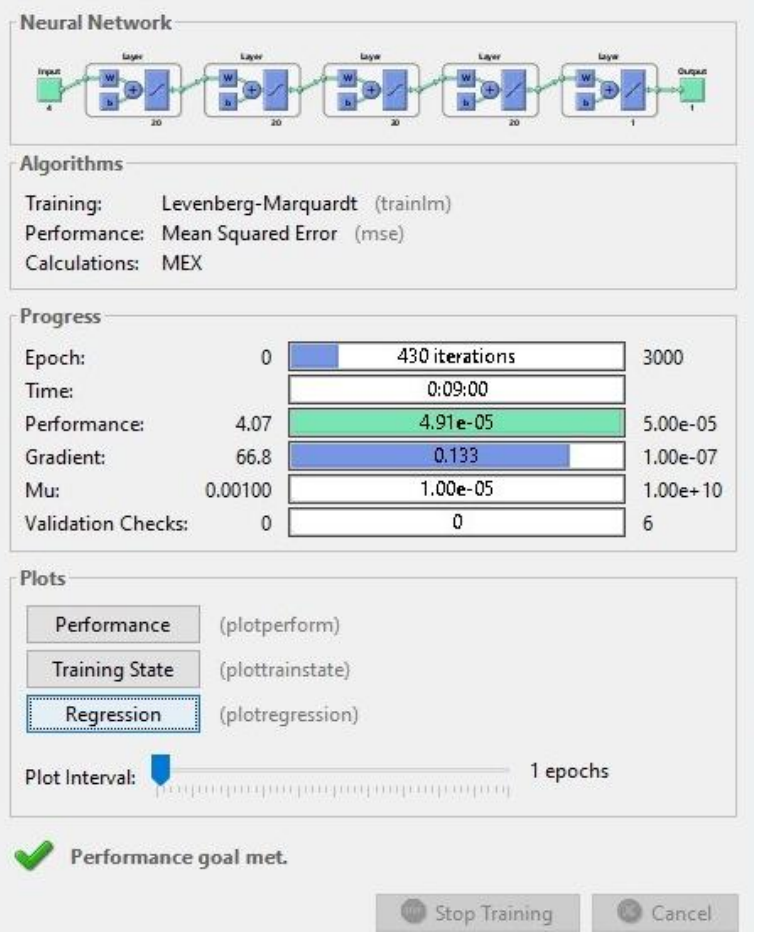

Fig 16: performance and training of (DTDNN) neural network

A Distributed Time-Delay Artificial Neural Network structure is used to reduce overshoot of speed. The 8023 sample from SMIB datasets are used for input data, samples were obtained from simulation of the real model of one generator in station with FPID-PSS and training it by Neural Network DTDNN . The DTDNN processes those given data to recognize and reduce overshoot of speed oscillation. Fig 17 shows the match of the training samples on the fit line and the target line to be achieved after training the neural network on the selected samples. Fig 18 illustrates the architecture and parameters used in simulation process.

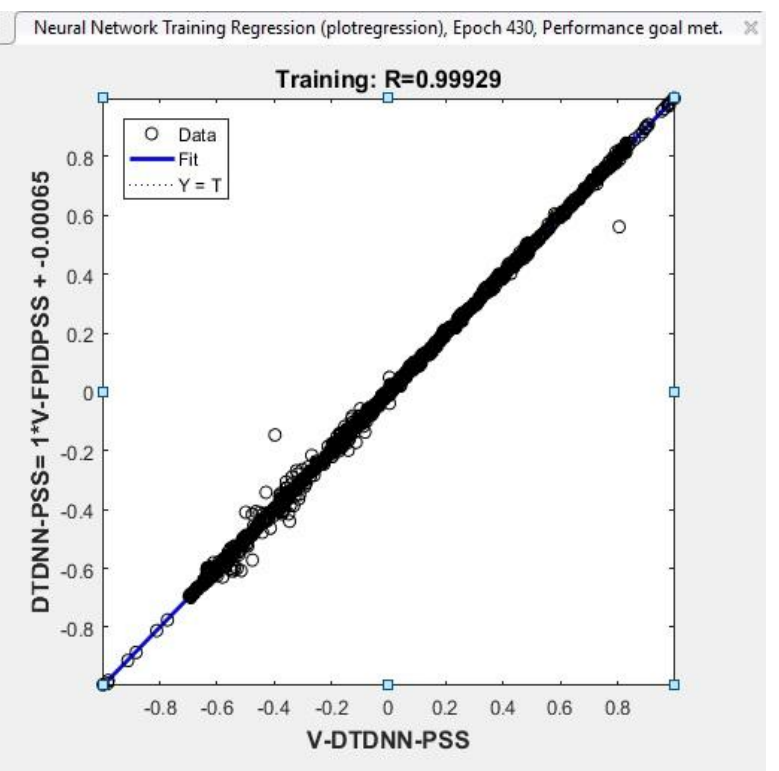

Fig 17: (DTDNN) Neural Network Training 


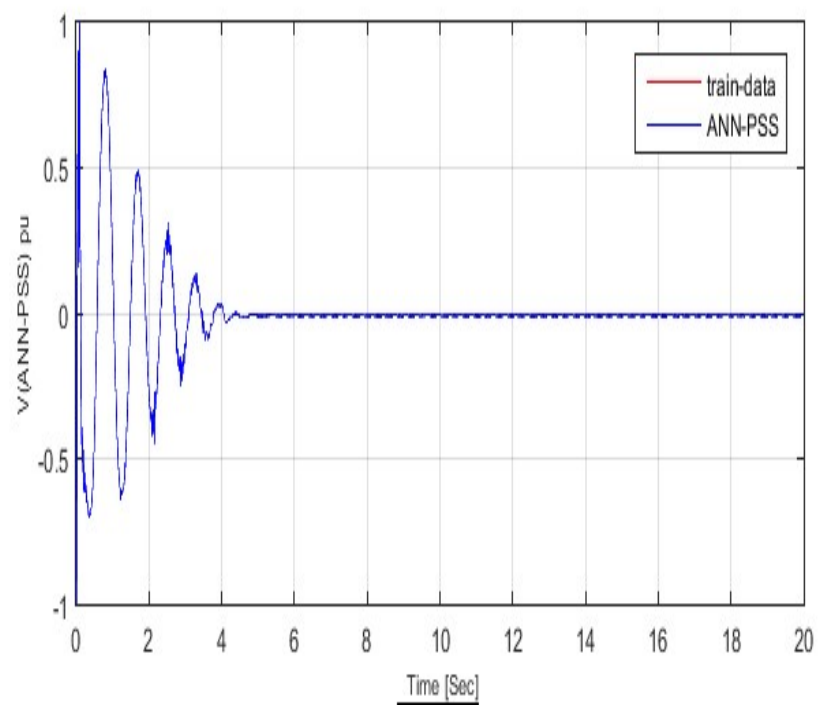

Fig 18: The (DTDNN) neural network output after training it on different samples

When the training process has done, DTDNN_PSS is installed to the system. The model of DTDNN_PSS is shown in Fig. 19

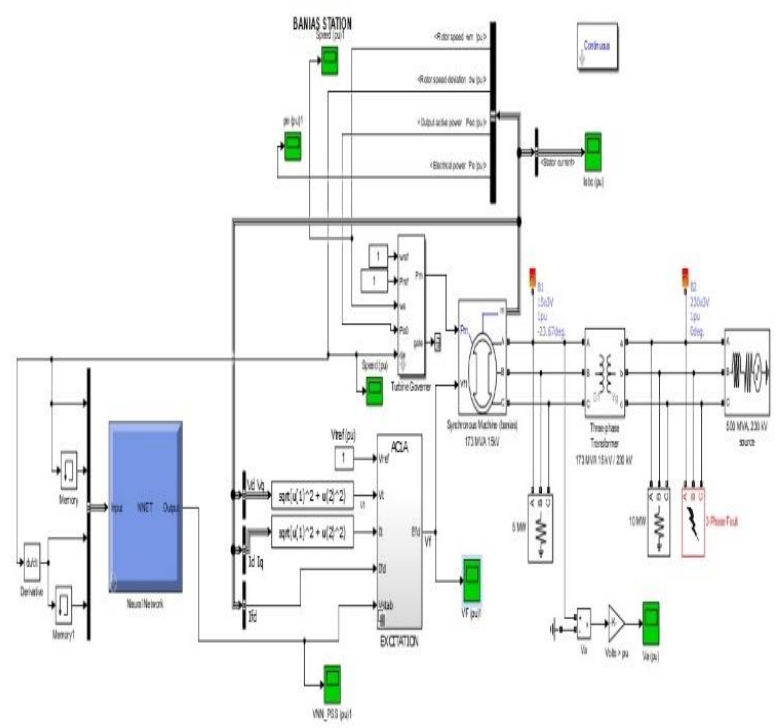

Fig 19: Structure of DTDNN_PSS and synchronous generator

\section{RESULTS AND DISCUSSIONS}

The model generated at the studied station with real parameters was simulated, where the results showed the unstability of the electrical system after three-phase faults. The electrical power changes (Pe) and output voltages of controllers (V-PSS) were studied for four cases: First case: Without using (PSS).

Second case: the dynamic response of the electrical system with (PSS).

Three case: Developing a PSS using (Fuzzy-PID) controller and studying the system's dynamic response.

Fourth case: The system's dynamic response after design (PSS) relies on an intelligent neural controller (DTDNN).

\subsection{The dynamic response of the electrical power for the four studied states.}

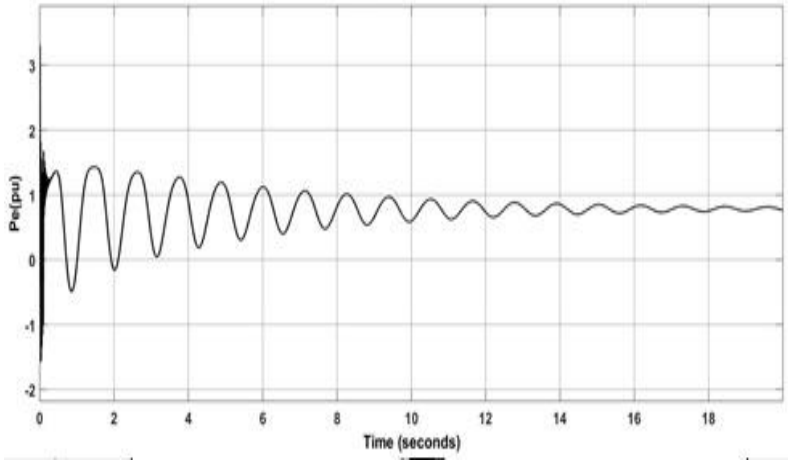

Fig 20: Dynamic responses of Pe without PSS

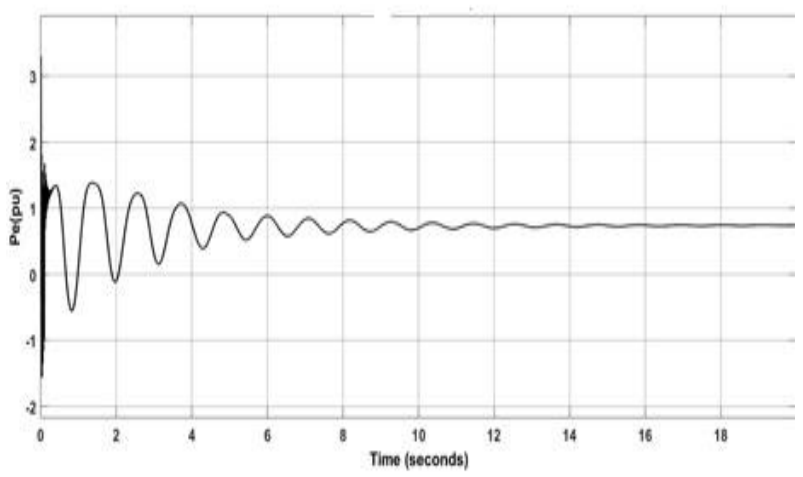

Fig 21: Dynamic responses of Pe with PSS

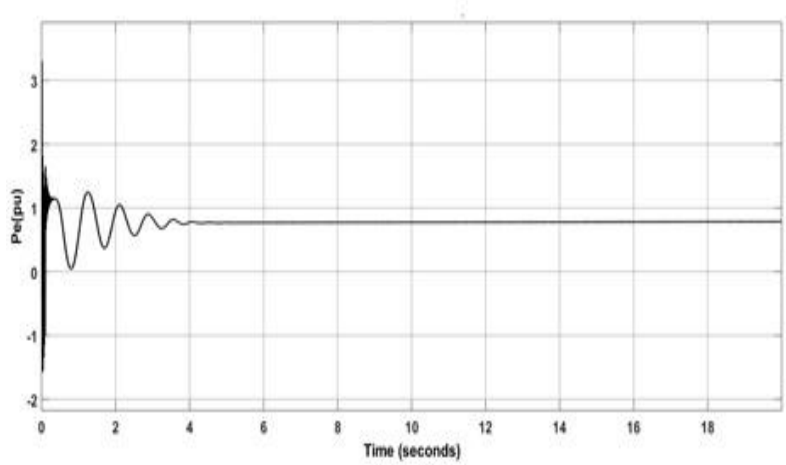

Fig 22: Dynamic responses of Pe with (Fuzzy-PID_PSS)

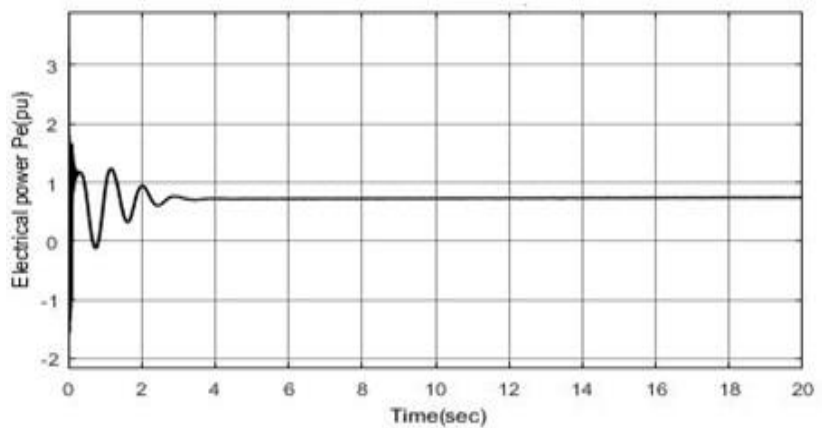

Fig 23: Dynamic responses of Pe with (DTDNN_PSS) 


\subsection{Output voltage changes for each (FL) and (Fuzzy-PID) and (DTDNN).}

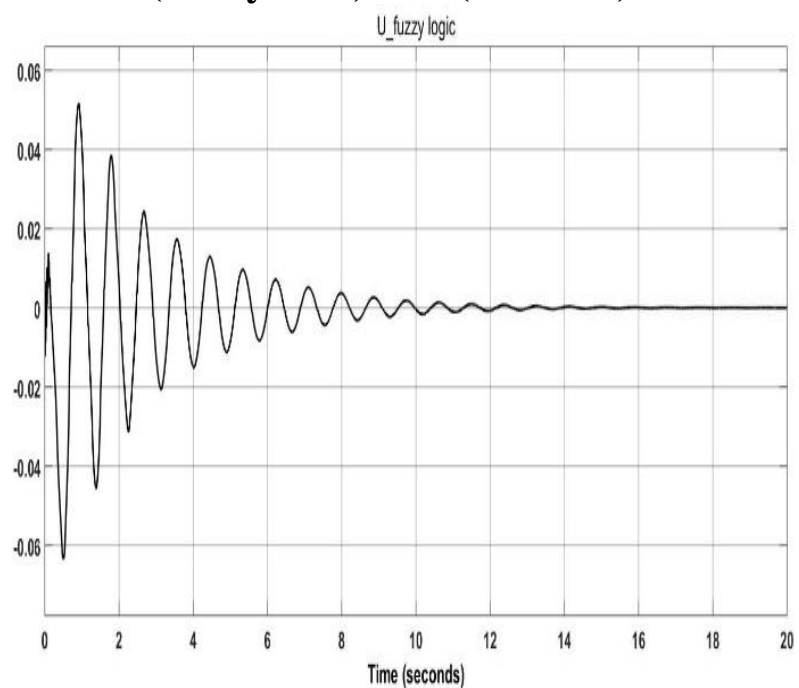

Fig 24: output voltage of (Fuzzy Logic) controller

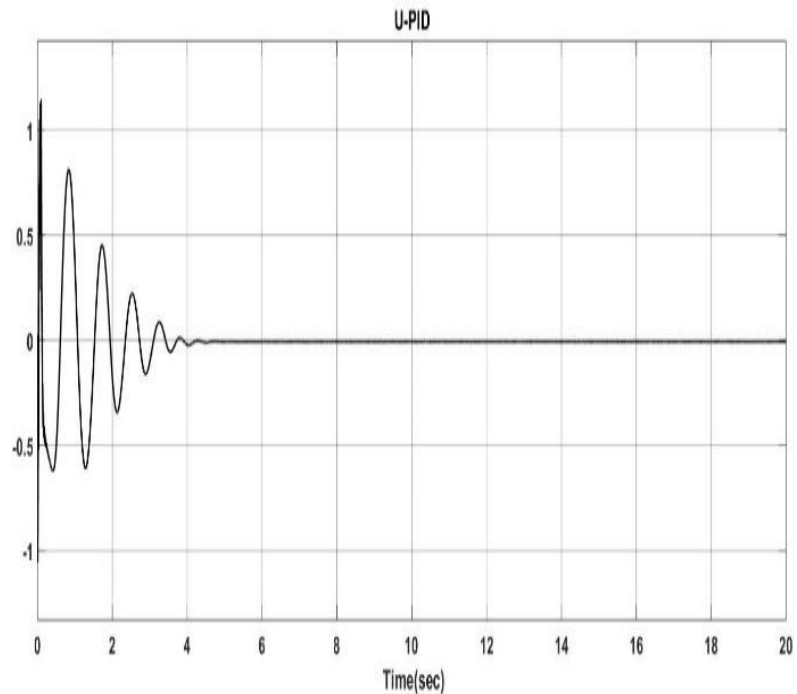

Fig 25: output voltage of (Fuzzy-PID_PSS) controller

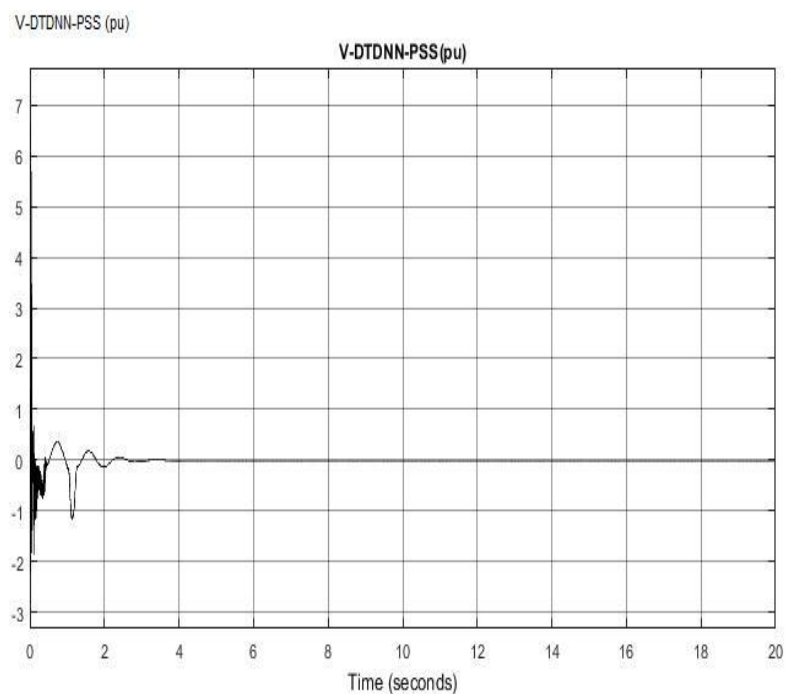

Fig 26: output voltage of (DTDNN_PSS) controller
Figures $20,21,23$ show the dynamic response of the electrical system after a three-phase fault and oscillations of electrical power, it has returned to stability after removing the a three-phase fault with a time of 30 seconds without using (PSS) while it stabilized with a time of 20 seconds with (PSS), but when using (Fuzzy-PID-PSS) the electrical system stabilized at a time of 4 seconds and the electrical power oscillations were reduced further than the previous two cases while when using (DTDNN-PSS) the electrical system stabilized at a time of 3 seconds and the electrical power oscillations were reduced More than previous cases .

Figures 24, 25, 26 show the dynamic response of the output voltage of each the Fuzzy Logic and PID and ANN-PSS controllers. the output voltage of the Fuzzy Logic controller stabilized at 14 seconds while the output voltage of the PID controller stabilized at 4 seconds while the output voltage of the DTDNN-PSS stabilized at 2 seconds.

\section{CONCLUSION}

This study proposes a modern control method (DTDNN-PSS) with an electrical system consisting of synchronous generator and a turbine connected to an infinite bus(SMIB), simulation results showed activity of the new neural stabilizer (DTDNNPSS) in damping oscillations of transient states and reaching stability in time Less compared to the conventional power system stabilizers (PSS) used in the studied station. A comparison study of the proposed (DTDNN-PSS) controller and coordinated (Fuzzy-PID) is carried out with conventional controllers such as PSS. The result observed by simulation showed that the (DTDNN-PSS) controller obtained a robustness performance for all the cases study.

\section{ACKNOWLEDGMENTS}

This Work was Supported by Department of Electrical engineering and Department of Artificial Intelligent, Faculty of Information Engineering and Faculty of Electrical \& Electronics Engineering, University of Aleppo/Syria.

\section{APPENDIX}

The parameter values of the power system:

$\mathrm{T} 1=0.15 \quad \mathrm{~T} 2=0.03 \quad \mathrm{~T} 3=0.15 \quad \mathrm{~T} 4=0.03 \quad \mathrm{~T} 10=0.15 \quad \mathrm{~T} 11=0.03$ Tw1=2 T6=0 T7=2 T8=0.3 T9=0.15 ks1=1 ks2=3 ks3=1 ks4=1 Tw4 $=\quad$ Tw3 $=2 \quad$ Tw2 $=2 \quad$ ks1 $=20 \quad \mathrm{~N}=1 \quad \mathrm{M}=5$ $\mathrm{SG}(\mathrm{MVA})=173 \quad \cos \varphi=0.8 \quad \mathrm{U}(\mathrm{kv})=15 \mathrm{H}=4.8 \mathrm{Xd}(\mathrm{pu})=1.3 \mathrm{Xd}$ ${ }^{\prime}(\mathrm{pu})=0.29 \quad \mathrm{Xq}^{\prime}(\mathrm{pu})=0.65 \quad \mathrm{Xd}(\mathrm{pu})=0.252 \quad \mathrm{Xq}(\mathrm{pu})=0.243$ Td'o(sec $)=1$ Tq'o(sec $)=1.25 \mathrm{~F}(\mathrm{HZ})=50 \mathrm{~N}(\mathrm{rpm})=1500$

\section{REFERENCES}

[1] Latha.r ,Kanthalakshmi.s and ,Kanagaraj- 2013, Design of power system stabilizer using fuzzy based sliding mode control technique.

[2] A. A gharaveisi , A.darabi , M.monadi , A KhagehZadeh,M.Rashidi-nejad,2005 ,Performance evaluation of an ANFIS based power system stabilizer applied in multimachine power system.

[3] Arun Kumar Sahu, Vishwanath Prasad Kurmi, 2017, Stability Enhancement of Power System using Fuzzy Logic based power system stabilizer international Journal of Science Engineering and Technology, Volume 5 Issue 3.

[4] Jenica Ileana Corcau, Eleonor Stoenescu, 2007, Fuzzy logic controller as a power system stabilizer, Issue 3, Volume 1.

[5] Hassan Bevrani, Takashi Hiyama, \&HosseinBevranic, 
2011-"Robust PID based power system stabiliser: Design and real-time implementation", Electrical Power and Energy Systems 33, pp 179-188.

[6] P. Kundur, 1994, Power System Stability and Control, MacGraw-Hill.

[7] M. Bounou. "Contribution a l'exploitation robuste des reseaux electriques par la methode de taguchi”. Thesis, Polytechnique, Montréal. 1997.

[8] Ang, K.H., Chong, G. and Li, Y. (2005) PID Control System Analysis, Design, and Technology.

[9] Astrom, K.J. and Hägglund, T. (1995) PID Controllers:
Theory, Design, and Tuning. Research Triangle Park, NC: Instrument Soc. Amer.

[10] G., Magdy. (2016) Digital Redesign of Analog Controllers for Power Systems Using PIM. LAP LAMERT-Academic Publishing, paperback, 164 Pages, 2016.

[11] El-Hawary, M.E. (1998) Electric Power Applications of Fuzzy System. IEEE Press.

[12] Baliyan A, Gaurav K \& Mishra SK (2015). A Review of Short Term Load Forecasting using Artificial Neural Network Models. Procedia Computer Science, 48, 121125 . 